NASA Contractor Report 4321

\title{
Micromechanical Model of Crack Growth in Fiber Reinforced Ceramics
}

Asher A. Rubinstein and Kang Xu

GRANT NAG.3-967

SEPTEMBER 1990

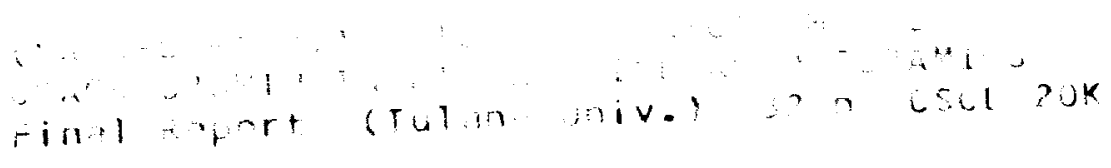




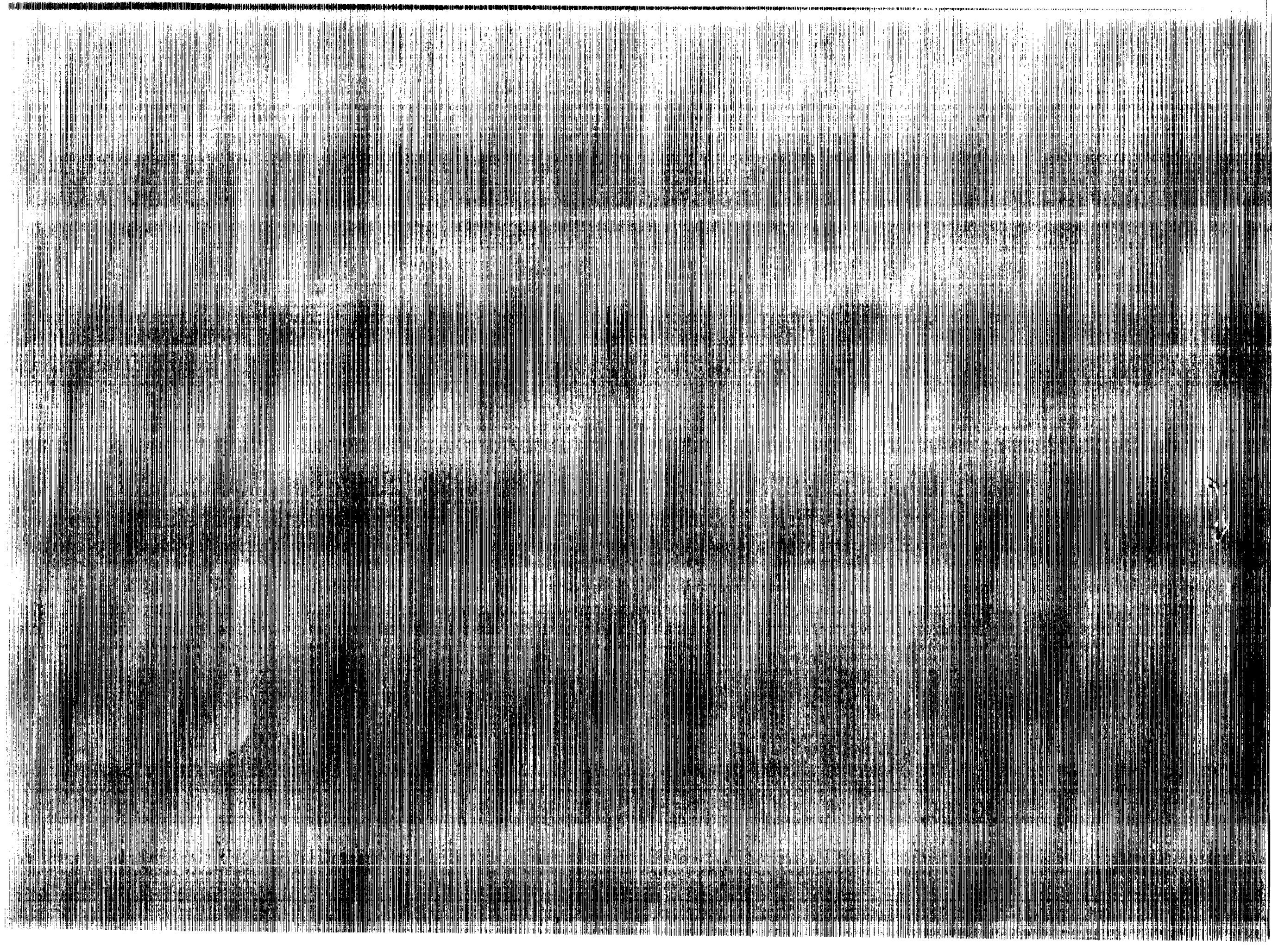


NASA Contractor Report 4321

\section{Micromechanical Model of Crack Growth in Fiber Reinforced Ceramics}

Asher A. Rubinstein and Kang Xu

Tulane University

New Orleans, Louisiana

Prepared for

Lewis Research Center

under Grant NAG3-96?

\section{N/SA \\ National Aeronautics and \\ Space Administration \\ Office of Management \\ Scientific and Technical \\ Information Division





\title{
MICROMECHANICAL MODEL OF CRACK GROWTH IN FIBER REINFORCED CERAMICS
}

\author{
Asher A. Rubinstein and Kang Xu \\ Department of Mechanical Engineering \\ Tulane University \\ New Orleans, LA 70118
}

\begin{abstract}
A model based on the micromechanical mechanism of crack growth resistance in fiber reinforced ceramics is presented. The formulation of the model is based on a small scale geometry of a macrocrack with a bridging zone, in this case the process zone, which governs the resistance mechanism. The effect of high toughness of the fibers in retardation of the crack advance, and the significance of the fiber pullout mechanism on the crack growth resistance, are reflected in this model. The model allows one to address issues such as influence of fiber spacing, fiber flexibility, and fiber-matrix friction.

Two approaches were used. One represents the fracture initiation and concentrates on the development of the first microcrack between fibers. An exact closed form solution has been obtained for this case. The second case deals with the development of an array of microcracks between fibers forming the bridging zone. An implicit exact solution is formed for this case. In both cases, a discrete fiber distribution is incorporated into the solution.
\end{abstract}

\section{INTRODUCTION}

Ceramic materials have promising potential for the atrospace industry as the structural materials of the future. A serious drawback in their application is their brittle pattern of failure. To improve the situation, material reinforcements are used in the form of additives, such as particles or fibers. The function of these additives is to trap the growing crack and, thus, to increase the toughness of the resulting composition. A rather detailed description of these additives with key references are given by Rose (1987). Mostly, these reinforcements improve fracture resistance under tensile loading. This paper deals with a 
case of ceramics reinforced by unidirectional long fibers. There are several aspects involved in the mechanics of fiber reinforcement. Two essentially different situations regarding the effect of fiber reinforcement must be pointed out. One case may be described as a long crack through the matrix with the fibers holding the crack surfaces (Aveston, Cooper and Kelly, 1971, Luh and Evans, 1987). The second case deals with a growing crack through the matrix and fibers with a region in the vicinity of the crack tip where the fibers are still intact. This paper deals with the second case only. The aim of this paper is to develop a theory describing the fracture resistance build up during this crack growth. The physical situation considered here is very similar to the case considered by Budiansky and Amazigo (1989), but the method of our analysis is totally different, and the results are different to a degree as well. Our formulation is based on the discrete fiber distribution, and it includes a restriction on fiber flexibility.

Several methods of analysis of fiber reinforcement have been developed and presented in the literature. The common feature of these methods is representation of the reinforcement effect of the fibers, or inclusions, as a continuous distribution of forces on the crack surfaces (McCartney 1987, Rose 1987, Nemat-Nasser and Hori 1987, Budiansky and Amazigo, 1988 and 1989). The distribution function of these forces may be specified (Nemat-Nasser and Hori 1987); or the relationship between the surface separation and the acting surface tractions may be assigned, and then the integral equation formed to find the resulting distribution function and other important parameters of the problem. The relationship between the local crack opening and acting surface tractions is the critical item of the analysis. In the above-cited references, this relationship is assumed to be linear, as it is assumed here, although we are not dealing with continuous distribution of surface tractions; rather, we relate the local crack opening to a net force on a fiber. The choice of this simplified linear relationship is motivated mostly by the fact that there are no experimentally established data which would specify a relationship for the fiber pullout under applied force. The formulation employed here may be easily generalized for a nonlinear relationship describing the fiber pullout.

As mentioned above, the failure process in fiber reinforced ceramics involves several aspects, some of which are not essential for the purpose of this study. We do not consider 
here fiber breaking (Marshal and Cox, 1987; Thouless and Evans, 1988), fiber - matrix interface debonding, and frictional effects modeled by Budiansky, Hutchinson and Evans, 1986.

Assumptions. We assume the elastic properties of the fibers to be very similar to the properties of the matrix with no significant difference in values of elastic constants. The difference between the strain magnitude in the fiber and in the matrix is insignificant at a finite distance from the crack surface, $y>0$ or $y<0$. The amount of fiber pullout is proportional to the net force acting on the fiber; that is, we assume a linear friction type law.

\section{FORMULATION AND ANALYSIS}

The proposed mathematical model of the analysis of the crack growing mechanism is based on consideration of a process zone development ahead of a macrocrack. The size of the process zone should be significantly smaller than the lergth of the crack, so the surrounding stress field is controlled by the stress intensity factor generated by the macrocrack. The main feature of the fracture process is the expansion of the main crack while some fibers remain intact. In two dimensions, this expansion appears as microcracks develop ahead of the macrocrack with bridges formed by the fibers. In our formulation, we consider a two dimensional problem corresponding to the described process. We consider the initial state of the crack growth, that is, formation of the first microcrack; the intermediate state; and a steady state case, when a complete assembly of microcracks starts to propagate without increasing the number of microcracks. The configurations of these problems are illustrated in Figure 1. and Figure 2. Assuming that elastic properties of the fibers are similar to the properties of the matrix, we treat the material as isotropic and homogeneous. We also neglect the difference in displacements between material points in the fibers and the matrix at any finite distance from the crack line $(y>0$ or $y<0)$. With these assumptions, the analysis described by Rubinstein, 1985 and 1987, can be applied. The basic relations of plane elastostatics in terms of analytic potentials are, Muskhelishvili, 1975:

$$
\begin{aligned}
& \sigma_{11}+\sigma_{22}=4 \operatorname{Re} \phi^{\prime}(z) \\
& \sigma_{22}-\sigma_{11}+2 i \sigma_{12}=2\left(\bar{z} \phi^{\prime}(z)+\psi^{\prime}(z)\right)
\end{aligned}
$$




$$
2 \mu\left(u_{1}+i u_{2}\right)=\kappa \phi(z)-z \overline{\phi^{\prime}(z)}-\overline{\psi(z)},
$$

here $\mu$ is a shear modulus, $\nu$ is a Poisson's ratio, and $\kappa=3-4 \nu$ for plane strain, or $\kappa=(3-\nu) /(1+\nu)$ for plane stress. Limiting our attention to the Mode I loading, so the direction of applied tension is parallel to the direction of fibers, the symmetry condition on $y=0$ can be stated as

$$
\sigma_{12}(z=x)=0=\operatorname{Im}\left(\bar{z}_{\phi}^{*}(z)+\psi^{\prime}(z)\right)
$$

Functions $\phi$ and $\psi$ are analytic in the plane with cuts along $y=0$, and, therefore, they may be considered as analytic in the upper half plane. Using condition (2.2) and applying the principle of analytical continuation, one obtains the relationship between the analytic potentials, which is true up to a real constant,

$$
\psi^{\prime}(z)=-z \phi^{*}(z) \text {. }
$$

The constant may be dropped since both sides of (2.3) have to vanish as $z \rightarrow \infty$. With relation ( .3 ), the expressions for the normal stress and displacement components along $z=x$ become

$$
\sigma_{22}=2 \operatorname{Re} \phi^{\prime}(x), \quad u_{2}=\frac{\kappa+1}{2 \mu} \operatorname{Im} \phi(x) .
$$

Thus, only one analytic function $\phi$ has to be determined, and the boundary conditions can be written in terms of this function. The condition at infinity states that function $\phi$ " has to match the applied stress field, which should be given in terms of a remote stress intensity factor $K_{\infty}$ (we consider Mode I loading only).

$$
\phi^{\prime}(z) \longrightarrow \frac{K_{\infty}}{2 \sqrt{2 \pi z}} \text { as } z \rightarrow \infty
$$

To form a correct boundary value problem we consider the upper half plane as a region for $\phi$; thus, to complete a set of boundary conditions the boundary values on $z=x$ have to be stated. On the intervals corresponding to the macrocrack and microcracks, the zero traction statement will be complete if in addition to the symmetry condition (2.2), we form a statement of zero normal stress given by (2.4). On the interval ahead of the complete assembly of the microcracks the displacement has to be zero, and, equivalently, its 
derivative can be stated as such. On the ligaments corresponding to the fibers, the displacement is not zero, due to the friction between the fibers and matrix. The matrix separates here, and the load is transmitted onto the fibers, and this is an essential aspect of the toughening mechanism which has to be accounted for. To realize the nature of the displacement on these ligaments, we consider the following:

It would be reasonable to assume the fibers to be in the form of circular cylinders, and therefore, the deformation created by the fiber pullout locally should remain of cylindrical symmetry. This means that the displacement of the matrix along the rim of the matrix-fiber interface has to be constant, if the direction of the pullout is perpendicular to the matrix surface. Therefore, in a two dimensional model, the displacement may be considered to be of constant magnitude on each interval corresponding to the separated matrix and the intact fiber. The value of this displacement is different for each particular interval, and it is controlled by the friction law which relates this displacement to a net force acting on the fiber. As a result, the derivative of the displacement on these intervals is zero; this completes the necessary set of boundary conditions for the function $\phi^{\prime}(z)$ in the upper half plane.

i) One link solution. The case of formation of the first microcrack can be resolved in closed form and, therefore, deserves a separate consideration. The geometry of this problem is given in Figure 1.

In addition to (2.5) the boundary conditions along $z=x$ are

$$
\begin{aligned}
& \operatorname{Re} \phi^{\prime}(x)=0 \text { on } x<0 \quad \text { and } a<x<b \\
& \operatorname{Im} \phi^{\prime}(x)=0 \text { on } 0<x<a \text { and } b<x
\end{aligned}
$$

The relationship between the force on the fiber $F$ and the matrix displacement $B$ is assumed to be linear, where $\lambda$ is a friction coefficient.

$$
\lambda F=B
$$

The assumption of the linear friction law is not necessary; as will be seen, the problem could be solved numerically for any nonlinear relationship instead of (2.7). The only reason we assume (2.7) is to simplify the problem, since in this case we have an exact closed form solution, and, additionally, currently there is no experimental evidence that the fiber pullout 
should be described differently.

The boundary conditions (2.5) and (2.6) form a mixed boundary value problem for an analytic function. Muskhelishvili, 1953, outlined the Keldysh-Sedov problem which deals with mixed problems for analytic functions, which are regular at $z \rightarrow \infty$. Rubinstein, 1985, used an equivalent approach to form a solution with a branch cut extended to infinity. It is clear that the same form of the analytic function will satisfy the conditions stated above. Thus, we have

$$
\phi^{\prime}(z)=\frac{K_{\infty}}{2 \sqrt{2 \pi}} \frac{z-d}{\sqrt{z(z-a)(z-b)}} .
$$

The constant $d$ should be determined from the equation (2.7). The force on the fiber is

$$
\begin{aligned}
& F=\int_{0}^{a} 2 \operatorname{Re} \frac{k_{\infty}}{2 \sqrt{2 \pi}} \frac{x-d}{\sqrt{x(x-a)(x-b)}} d x \\
& =2 \frac{K_{\infty}}{2 \sqrt{2 \pi}}\left[-2 \sqrt{b} K\left(\frac{a}{b}\right)+2 \sqrt{b} E\left[\frac{a}{b}\right]+d \frac{2}{\sqrt{b}} K\left(\frac{a}{b}\right)\right] .
\end{aligned}
$$

The following definitions of complete elliptic integrals were used here, Abramowitz, 1972:

$$
\begin{aligned}
& K(m)=\int_{0}^{1} \frac{d x}{\frac{\left(1-t^{2}\right)\left(1-m t^{2}\right)}{(m)}=\int_{0}^{1} \sqrt{\frac{1-m t^{2}}{1-t^{2}}} d x}
\end{aligned}
$$

The displacement of the matrix on the ligament corresponding to the intact fiber is found as a displacement gained due to the microcrack opening. Thus,

$$
\begin{aligned}
& B=2 \frac{\kappa+1}{2 \mu} \int_{b}^{a} \operatorname{Im} \frac{K_{\infty}}{2 \sqrt{2 \pi}} \frac{x-d}{\sqrt{x(x-a)(x-b)}} d x \\
& =\frac{\kappa+1}{\mu} \frac{K_{\infty}}{2 \sqrt{2 \pi}}\left[2 \sqrt{b} E\left[1-\frac{a}{b}\right]-d \frac{2}{\sqrt{b}} K\left[1-\frac{a}{b}\right)\right] .
\end{aligned}
$$

Introduce dimensionless constant 


$$
\Lambda=2 \frac{\lambda \mu}{\kappa+1}
$$

and from equation (2.7) find

$$
\frac{\mathrm{d}}{\mathrm{b}}=\frac{E\left[1-\frac{\mathrm{a}}{\mathrm{b}}\right)+\Lambda\left[K\left(\frac{\mathrm{a}}{\mathrm{b}}\right)-\mathrm{E}\left(\frac{\mathrm{a}}{\mathrm{b}}\right)\right]}{\mathrm{K}\left(1-\frac{\mathrm{a}}{\mathrm{b}}\right]+\Lambda \cdot K\left(\frac{\mathrm{a}}{\mathrm{b}}\right)} .
$$

The final expression for the force per unit thickness acting on the first fiber cell becomes

$$
F=K_{\infty} \sqrt{\frac{b}{2 \pi}} \frac{\pi}{K\left[1-\frac{a}{b}\right]+\Lambda \cdot K\left(\frac{a}{b}\right]} .
$$

The stress intensity factor is determined by evaluating the appropriate limits in the usual fashion.

$$
\begin{aligned}
& K_{0}=K_{\infty} \frac{d}{\sqrt{a b}} \\
& K_{a}=K_{\infty} \frac{d-a}{\sqrt{a(b-a)}} \\
& K_{b}=K_{\infty} \frac{b-d}{\sqrt{b(b-a)}}
\end{aligned}
$$

Substituting $\Lambda=0$ into equation (2.12), and then using this result in $(2.14,2.15$, and 2.16 ), one will obtain expressions given by Rubinstein, 1985, for a macrocrack interacting with a microcrack; unfortunately, the expression corresponding to (2.15) was misprinted in that reference.

The dimensionless parameter $\Lambda$ characterizes the matrix-fiber friction, or it can be interpreted as a spring constant (inverse to a standard definition), if the region $0<x<a$ is connected by a linear spring. The definition of this parameter is chosen in the form (2.7), so the limit case of no friction would correspond to $\Lambda:=0$, and a less constrained (softer) system will have higher values of $\Lambda$.

ii) The bridging zone of arbitrary length. The formulation described above is extended to the case of an arbitrary number of microcracks (say $N$ ) formed in the bridging zone. The 
geometry of this general case is given in Figure 2. The symmetry condition (2.2) with results (2.3) and (2.4) still apples. As mentioned previously, the displacement on the ligaments corresponding to fibers are assumed constant (different on each interval, of course). Thus the boundary conditions along the bridging zone become

$$
\begin{aligned}
& \operatorname{Re} \phi^{\prime}(x)=0 \text { on } x<0 \quad \text { and } a+p k<x<p(k+1) \\
& \operatorname{Im} \phi^{\prime}(x)=0 \text { on } p k<x<a+p k \text { and } p N<x, k=0,1,2, \ldots, N-1 \\
& k=0,1,2, \ldots, N-1
\end{aligned}
$$

In combination with asymptotic behavior (2.5) conditions (2.17) determine a general form of an analytic function up to $N$ constants. As is known (Muskhelishvili, 1972), there are several possibilities to form this function, which differ in the choice of the location of the singular points. A physically suitable choice is the case with singularities at crack tips. Thus, the stress potential $\phi^{\prime}(z)$ can be written as follows:

$$
\phi^{\prime}(z)=\frac{\mathrm{k}_{\infty}}{2 \sqrt{2 \pi}} \frac{\prod_{k=0}^{N-1}\left(z-\mathrm{d}_{k}\right)}{\sqrt{z \prod_{k=0}^{N-1}(z-a-p k)(z-p(k+1))}} .
$$

$N$ constants $d_{\mathrm{k}}$ have to be determined from the conditions on the fibers. Condition (2.7) becomes

$$
\lambda F_{k}=B_{k^{\prime}} \quad k=0,1,2, \ldots, N-1
$$

where $B_{\mathrm{k}}$ is the cumulative displacement on the interval on the left of the microcrack $k$; so $k=0$ corresponds to the location of the first fiber. Denoting $\Delta \mathrm{B}_{\mathrm{i}}$ the displacement gain due to nonuniform opening of the microcrack $i$, the total displacement on the interval $k$ is

$$
\mathrm{B}_{k}=\sum_{i=k}^{N-1} \Delta \mathrm{B}_{i} .
$$

The value of the displacement increment gained over the microcrack $k$ is determined as 
integral

$$
\begin{aligned}
& \Delta B_{k}=\frac{\kappa+1}{\mu} \int_{p(k+1)}^{a+p k} \operatorname{Im} \phi^{\prime}(x) d x= \\
& =\int_{a+p k}^{p(k+1)} \frac{(k+1) k_{\infty} \prod_{2 \mu 0}^{N-1}\left(x-d_{i}\right)}{2 \pi x(x-a-p k)(p k+p-x) \prod_{j=0, j \neq k}^{N-1}(x-a-p j)(x-p j-p)} \\
& =
\end{aligned}
$$

The force in (2.19) is obtained by integration of the real part of the stress function; thus,

$$
F_{k}=2 \int_{p k}^{a+p k} \operatorname{Re}^{\prime}(x) d x=2 \int_{p k}^{a+p k} \frac{k_{\infty} \prod_{i=0}^{N-1}\left(x-d_{i}\right)}{2 \sqrt{2 \pi x \prod_{j=0}^{N-1}(x-a-p j)(x-p j-p)}}
$$

$N$ equations $(2.19)$ with equations $(2.20,-22)$ form a complete system for determination of the $N$ constants $d_{\mathrm{k}}$. This system is highly nonlinear. We solve this system by using the following observation.

Rewrite the products in the numerators of (2.21) and (2.22) into polynomials

$$
\prod_{k=0}^{N-1}\left(x-d_{k}\right)=\sum_{i=0}^{N} c_{i} x^{i}, \quad c_{N}=1
$$

Substituting (2.23) into (2.21) and (2.22), taking coefficients $c_{\mathrm{i}}$ out of the integrals, and substituting the results into equations (2.19) one obtains a linear system for the coefficients $c_{\mathrm{i}}$. On the other hand, the roots of the algebraic equation

$$
\sum_{i=0}^{N} c_{i} x^{i}=0
$$

are the constants $d_{\mathrm{k}}$ that we needed to find. Thus the problem is reduced to the numerical 
solution of the algebraic equation of power $N$. Usually the most difficult part of the solution is the location of the roots. In the case considered here, this is not a problem; the value of the constant $d_{\mathrm{k}}$ corresponds to the location of the maximal opening of the microcrack number $k$. Thus, the intervals of the location of the roots of the equation (2.24) are completely determined, and this equation can be solved numerically with any a priori specified accuracy. As a practical matter, the constants $d_{\mathrm{k}}$ are necessary only for verification of the resulting stress function (2.18); for any other purpose the substitution (2.23) may be used. Thus, the problem is reduced to a linear system.

A detailed numerical scheme of this solution of the system (2.19) and the integration procedure are given in the appendix.

The stress intensity factors at the crack tips are determined by taking the appropriate limits, so

$$
\begin{aligned}
& K_{0}=K_{\infty} \frac{\prod_{k=0}^{N-1} d_{k}}{\sqrt{p^{N} N ! \prod_{k=0}^{N-1}(a-p k)}}, \\
& k_{a+p i}=k_{\infty} \frac{\prod_{k=0}^{N-1}\left(a+p i-d_{k}\right)}{\sqrt{(a+p i)(p-a) \prod_{k=0, k \neq i}^{N-1} p(i-k)(a-p(k+1-i))}},
\end{aligned}
$$

$$
K_{p i}=K_{\infty} \frac{\prod_{k=0}^{N-1}\left(p i-d_{k}\right)}{\sqrt[p i(p-a) \prod_{k=0, k \neq i+1}^{N-1} p(i-k+1)(p(i-k)-a)]{ }} .
$$

$i=1,2, \ldots, N$

The integrals (2.25-27), as well as the integrals described in appendix, were evaluated by using Gaus - Chebyshev numerical procedure. 


\section{RESULTS}

The numerical data given in this section were obtained in accordance with the following interpretations of the physical effects taking place during the process of the crack growth. The main factors to consider are the crack growth resistance of the composite, that is material resistance build up due to extension of the bridging zone, and the limiting value of the length of the bridging zone. The resistance, or toughening, of the composite is determined by the change of the local stress intensity factor acting on the uncracked matrix at the leading crack tip (at $x=N p$ ), due to extension of the bridging zone. The maximal length of the bridging zone and, therefore, the maximal toughening, is determined by the strength of the fibers, and, as a result, is controlled by the net force acting on the first fiber (counting from the main crack tip, as in the Figure 2.) or by the local stress intensity factor $K(0)$. The behavior of these parameters within the bridging zone determines the stability of the zone development.

The initial development of the bridging zone has been analyzed on the basis of the one link model. Figure 3. depicts a dependence of the leading stress intensity factor (relative value with respect to remote value is given) acting on the uncracked matrix versus the coefficient $\triangle$ and the relative fiber thickness. In the sarne figure the topographical map of this stress intensity distribution is given. The data are given for the ratio $a / b$ ranging from 0.05 to 0.95 , and values of $\triangle$ ranging from 0.0 to 3.0. The choice of the range of values of $\Lambda$ is arbitrary since no data were available to relate it to a practical material composition. The natural limitation on this value that we used, is a requirement that the net force on the fiber remain strictly positive. That is, the displacement produced by the fiber slip out should not reach the crack opening displacement which would take place without fiber restraint. The physical nature of the bridging effect is well illustrated here. Increasing values of $\Lambda$ correspond to an easier fiber pullout, and accordingly, with higher fiber pullout, the load gets transmitted from the main crack onto the leading crack tip at $x=b$. The data given in Figure 3. correspond to a continuous growth of the microcrack ahead of the fiber, as well. One notes that a small microcrack in combination with high values of $\Lambda$ leads to unexpected high values of the leading stress intensity factor. This effect is due to the enforcement of a constant crack opening displacement on the matrix-fijer intersection. The reality of this 
condition depends on the flexibility of the fiber, but it is evident that for any fiber there is a critical length of a microcrack when this effect will take place. Below this critical length, the smaller microcrack will experience a higher value of the stress intensity, and that value will decrease with the microcrack extension until it drops below the critical value. This effect is taking place only after the parameter $\triangle$ reaches a certain value. As follows from the shape of the surface in Figure 3., smaller values of $\Lambda$ will allow stable existence of a small microcrack. This peculiar dependence of $K(b)$ on the microcrack size will take place as well in the case of a relaxed condition on the displacement on the matrix-fiber interface. For example, our preliminary analysis shows the presence of the same effect in the case when this displacement is specified as a linear function this displacement is specified as a linear function across the fiber. Physically, the restriction imposed here represents the fiber flexibility, which restrains the crack surface shape and, therefore, is equivalent to an additional moment applied in the vicinity of the crack tip.

The net force acting on the fiber with the corresponding topographical map are given in Figure 4, and the stress intensity factor on the main crack is given in Figure 5. The value of the net force on the fiber is given as a value per unit thickness normalized by the value of the net force acting on the fiber prior to matrix cracking. The shape of the fiber cross-section is not considered; thus, assuming that the fiber is represented by the entire ligament, the dimensionless force in Figure 4. is given as

$$
F^{*}=\frac{\pi}{2} \sqrt{\frac{b}{a}} \frac{1}{k\left[1-\frac{a}{b}\right]+\Lambda k\left[\frac{a}{b}\right]} .
$$

The stress intensity factor in Figure 5. is normalized by the applied stress intensity factor. The data in Figure 5. show the existence of the optimal fiber spacing - parameter $\wedge$ combination for lower values of $K(0)$. In Figure 6., data corresponding to the leading edge of the fiber ( $x=a$, Figure 1.) are given. The stress intensity factor at this point decreases with increasing value $\Lambda$, that is, with decreasing stiffness of the composite. This stress intensity factor becomes negative, which indicates that the tensile stress singularity becomes eliminated and the stress state changes into compression. The curve of $K(a)=0$ on the topographical map in Figure 6. appears to coincide with the optimal path for minimal values 
of $K(0)$, Figure 5, and an optimal path on the topographical map in Figure 3. The existence of the stress singularities at point $x=0$ and $x=a$ may be argued as physically not very realistic. However, in terms of the presented formulation which has no room for the fiber-matrix delamination along the fiber direction, these stress intensity factors may be used as a quantitative characteristic of the potential delamination.

The development of fracture parameters during an extension of the bridging zone is discussed below. The length of the bridging zone is represented in terms of the number of microcracks developed within the zone. The algorithm given in the previous section may be used for any length of the bridging zone. However, the maximum number of microcracks within the bridging zone considered here is limited by the applicability of the small scale model. The small scale model may be applied up to a point, when the influence of non singular terms in the surrounding stress field becomes significant, and the value of the leading stress intensity factor no longer may be determined on the basis of the applied stress intensity factor only. An additional limitation of the length of the bridging zone in the small scale formulation is related to a specific feature of this model, and associated with the following fact: When the leading stress intensity factor is significantly reduced with respect to a value acting at the tip of the main crack, the insignificant amount of fiber pullout in the area of a leading crack tip brings that ligament surface to a state close to traction free position. In other words, the local stress state becomes so released that the effect of fiber reinforcement becomes insignificant. The data given by Rubinstein, 1985, for the case of the array of microcracks ahead of the macrocrack, may be used to estimate a reasonable length of the bridging zone with regard to the applicatility of the small scale model. The second factor in establishing effectiveness of the fiber reinforcement was evaluated computationally. We found that a bridging zone with eighteen microcracks may serve as a reasonable representation of the major effects taking place in association with the bridging mechanism.

The physical limitation for the extension of the bridging zone is the strength of the fibers. The first fiber (counting from the main crack tip) bears the highest load and supposedly limits the total length of the bridging zone. In Figure 7. a history of the force increase during the bridging zone extension is given. We considered three cases here, 
$a / p=0.25, a / p=0.5$ and $a / p=0.75$, which reflect major tendencies in the model. The length of the bridging zone is given in terms of a number of microcracks, $N$, involved in the zone. The parameter $\wedge$ was varying from 0 to 3.5 in 0.5 intervals. The net force acting on the fiber was computed in accordance with equation (2.22) and then normalized by the net force acting on the ligament corresponding to a first fiber prior matrix cracking. That is

$$
\mathrm{T}_{1}(\mathrm{~N}>0)=\frac{1}{2 \mathrm{~K}_{\infty}} \sqrt{\frac{2 \pi}{\mathrm{a}}} \mathrm{F}_{1} \text {. }
$$

In the case $N=0$ the matrix is still intact, and the net force on the fiber is a portion of the force on the ligament proportional to the area occupied by the fiber. Assuming that the fiber has a circular cross section, and that the period of fiber distribution is the same in the crack front direction, the force on the first fiber is

$$
\mathrm{T}_{1}(\mathrm{~N}=0)=\frac{\pi \mathrm{a}}{4 \mathrm{p}} \frac{1}{2 \mathrm{~K}_{\infty}} \sqrt{\frac{2 \pi}{\mathrm{a}}} \mathrm{F}_{1} .
$$

The data in Figure 7. shows that after a relatively small number of microcracks developed in the bridging zone, the value of the force acting on the first fiber reaches the maximum, and consequently this value does not depend on an additional number of microcracks. Higher values of the parameter $\Lambda$ increases the length of this transition. Thus, the assumption that the maximal value of the force on the first fiber bounds the length of the bridging zone, is not well justified. Consequently, the maximal amount of fiber pull out, as a limiting criterion for the bridging zone length, has to be ruled out in terms of this model inasmuch as that amount is proportional to the magnitude of the force and, therefore, has a similar behavior with respect to the length of the bridging zone.

A similar trend is observed in the behavior of the stress intensity factor acting at the tip of the main crack, Figure 8 . This stress intensity factor also characterizes the stress intensity within the fiber, and, additionally, it can be used as a parameter determining the possibility of delamination at the fiber matrix interface. As shown in Figure 8., the values of the stress intensity factors reach their maximum after relatively few microcracks develop in the bridging zone. As in the case of the force on the first fiber, the higher values of the parameter $\Lambda$ increase the length of the transitional bridging zone, after which the stress intensity factor practically does not change. 
The distribution of the forces acting on the fibers and the local stress intensity factors (only stress intensity factors at the leading tips are represented) are given in Figure 9. The values of force in Figure 9a. are normalized differently from the previous case. If in the previous case the fiber was assumed to be of constant thickness and the fiber spacing was changed, the force in Figure 9a. is given as a force per the period of fiber spacing. That is

$$
\mathrm{T}_{\mathrm{k}}=\frac{1}{2 \mathrm{~K}_{\infty}} \sqrt{\frac{2 \pi}{\mathrm{p}}} \mathrm{F}_{\mathrm{k}} \text {. }
$$

The subscript $k$ in (3.4) indicates the position of the fiber. In this form the force on the fiber practically does not depend on the fiber spacing ratio, and insignificantly depends on the parameter $\Lambda$ in the leading portion of the bridging zone.

The distributions of the leading stress intensity factors for different spacing ratios are given in Figures 9b, 9c, and 9d. No significant dependence of the local stress intensity factors on the parameter $\wedge$ are observed here, except for the stress intensities in the vicinity of the main crack tip (first few microcracks) and the stress intensity factor at the leading crack tip $(x=N p)$. Higher values of $\wedge$ produce higher stress intensity at the leading crack tip and lower stress intensity at the main crack tip. Basically, the fiber pull out mechanism is the mechanism of load redistribution from the main crack onto the array and in a greater part onto the leading microcrack. This phenomenon is reflected in the resistance curves given in Figure 10. The resistance curves represent material ability to sustain higher load due to internal toughening mechanisms. The composite fracture resistance is measured as a value inverse to the leading stress intensity factor $\left(R=K_{\infty} / K(N p)\right)$ and is given versus the length of the bridging zone in terms of the number of microcracks. Three cases are given in Figure 10, for different spacing ratios, and, additionally, the dependence of the composite resistance on the fiber spacing ratio $a / p$ is given here for the incremental values of $\Lambda$. The fracture resistance increases with extension of the bridgng zone, while the higher resistance is typical for lower values of the parameter $\Lambda$. The resistance curves obtained here differ from the curves given by Budiansky and Amazigo, 1989; the most significant difference of our results as compared with the data from this reference is in the spread of our curves with respect to the values of $\Lambda$. The relationship used by Budiansky and Amazigo for the force on the fiber and the local displacement are similar to one used here, but the measure of the 
bridging zone length is different in our case. We were not able to convert the length scale used by Budiansky and Amazigo, 1989, to our case.

The sensitivity of the resistance process to the fibers spacing ratio $a / p$ is quite significant. There are optimal values of the $a / p$ ratio for the corresponding values of the pullout parameter $\Lambda$, when the fiber reinforcement is most effective. The tendencies of the resistance curves show that higher resistance values may be obtained with an increase of the bridging zone. However, the applicability of the small scale model becomes questionable for a large bridging zone. The leading stress intensity factors computed using the small scale approach are too small, and, therefore, the contribution from the higher order terms in the Williams expansion may no longer be neglected.

\section{CONCLUSIONS}

A closed form exact solution was obtained for a single link model characterizing an initiation of the bridging zone development.

An additional toughening, or anti-toughening (depending on $a / p$ ratio) aspect was ooserved, that is, constraint on the rotation of the matrix in the bridging zone due to the limited fiber flexibility.

An implicit exact solution was formed for an extended bridging zone of arbitrary length. The solutions presented here take into account the discrete distribution of the fibers.

The higher values of the parameter $\Lambda$, corresponding to a reduced fiber pullout resistance, reduce fracture resistance build-up by transforming the load onto the leading crack tip. On the other hand, in the case of high values of $\Lambda$, the tension on the trailing fiber, that is, on the fiber holding the main crack, is reduced. Thus, as a result, lower fiber pullout resistance will produce a long and stable bridging zone. In contrast, high fiber pullout resistance, that is, lower values of $\Lambda$, will concentrate the load on a small region in the vicinity of the main crack, maintaining high tension on the fiber at the main crack tip and, thus, creating a higher possibility of failure of this fiber, so the bridging zone will have a tendency to propagate as a whole.

There is an optimal combination of fiber pullout resistance and fiber spacing when the highest fracture resistance may be achieved for the same length of the bridging zone. 
Following the data given in Figure 10b, the optimal fibers spacing ratio varies from $0.37(\Lambda=3.5)$ to $0.8(\Lambda=0.5)$.

\section{ACKNOWLEDGMENT}

This work was supported by NASA Lewis Research Center under Grant NAG 3-967.

\section{REFERENCES}

Abramowitz, M.

Stegun, A.

Aveston, J.,

Cooper, G.A.

Kelly, A.

Budiansky, B.

Hutchinson, J.W.

Evans, A.G.

Budiansky, B.

Amazigo, J. C.

Budiansky, B.

Amazigo, J. C.

Luh, E.Y.,

Evance, A.G.

Nemat-Nasser, $\mathbf{S}$.

Hori, $M$.

Marshall, D.B.

Cox, B.N.

McCartney, L.N.

Muskhelishvili, N.I. 1963

Muskhelishvili, N.I. 1972

Rose, L.R.F.

Rubinstein, A.A.

Rubinstein, A.A.

Thouless, M.D.

Evans, A.G.
1972 Handbook of Mathematical Functions, Dover, New York.

1971 Conference on The Properties of Fiber Composites, National Physical Laboratory, Guildford, Surrey: ICP Science and Technology Press. pp. 15-26

1986 J. Mech. Phys. Solids, 34, 167-189.

$1988 \quad$ J.Mech. Phys. Solids, 36, 167-187.

1989 J.Mech. Phys. Solids, 37, 93-109.

1987 J. Am. Ceram. Soc., 70, 466-69.

1987 Mechanics of Materia's, 6, 245-269.

1987 Acta Metall., 35, 2607-2619.

1987 Proc. R. Soc. Lond. A, 409. 329-350.

Some Basic Problems of the Theory of Elasticity, Noordhoff, Groningen, Holland.

Singular Integral Equations, Noordhoff, Groningen, Holland.

1987 J. Mech. Phys. Solids, 35, 383-405.

1985 International Journal of Fracture, 27, 113-119.

1987 Engineering Fracture Mechanics, 26, 15-21

1988 Acta Metallurgica, 36, 517-522. 
Coefficients of the algebraic equation (2.24) are found by solving a system of linear equations (A5), which is obtained as follows. Introduce notations

$$
G_{k i}=\int_{a+p k}^{p(k+1)} \frac{x^{i}}{\sqrt{x(x-a-p k)(p k+p-x) \prod_{j=0, j \neq k}^{N-1}(x-a-p j)(x-p j-p)}} d x
$$

and

$$
H_{k i}=\int_{p k}^{a+p k} \frac{x^{i}}{\sqrt{x \prod_{j=0}^{N-1}(x-a-p j)(x-p j-p)}} d x .
$$

Form a matrix

$$
A_{k i}=\Lambda H_{k i}+\sum_{m=k}^{N-1} G_{m i}, \quad k, i=0,1,2, \ldots, N-1
$$

and an array

$$
D_{k}=-\Lambda H_{k N}-\sum_{m=k}^{N-1} G_{m N}
$$

then equations (2.22) becomes

$$
\sum_{i=0}^{N-1} A_{k i} C_{i}=D_{k}, \quad k=0,1,2, \ldots, N-1 .
$$

The array (A4) emphasizes the fact that $C_{N}$ is known, and it is equal to one.

The coefficients $C_{i}$ of the algebraic equation (2.24) can be found by solving the system (A5), and then constants $d_{i}$ are determined by employing a simple numerical procedure for solution of a nonlinear equation with roots located on a specified interval. 
The integrals (A1) and (A2) are evaluated by using Gaus-Chebyshev quadrature. This numerical procedure is accurate up to the order of $4 \mathrm{M}$, where $\mathrm{M}$ is a number of nodes on the integration interval. The distribution of the nodes coincides with roots of Chebyshev polynomials of first kind. Thus, using Hermite's formulae for Chebyshev polynomials, we have

$$
H_{k i}=\frac{\pi}{M} \sum_{s=1}^{M} \frac{x_{s ;}^{j}}{\sqrt{x_{s}\left(x_{s}-p k-p\right)\left(x_{s}-a-p(k+2)\right) \prod_{\substack{j=0 \\ j \neq k, j \neq k+1}}^{N-1}\left(x_{s}-a-p j\right)\left(x_{s}-p j-p\right)}}
$$

with

$$
\mathrm{x}_{\mathrm{s}}=\mathrm{pk}+\frac{\mathrm{a}}{2}\left(\cos \frac{2 \mathrm{~s}-1}{2 \mathrm{M}} \pi+1\right)
$$

and

$$
G_{k i}=\frac{\pi}{M} \sum_{s=1}^{M} \frac{x_{s}^{i}}{\sqrt{x_{\substack{j=0 \\ j \neq k}}^{N-1}\left(x_{s}-a-p j\right)(x-p j-p) s}}
$$

with

$$
x_{s}=a+p k+\frac{p-a}{2}\left(\cos \frac{2 s-1}{2 M} \pi+1\right)
$$

Square roots in (A6) and (A8) have to be taken with consideration of the branch cut. The choice of the branch is based on the condition, that for $x>p N$ the result should be positive. 


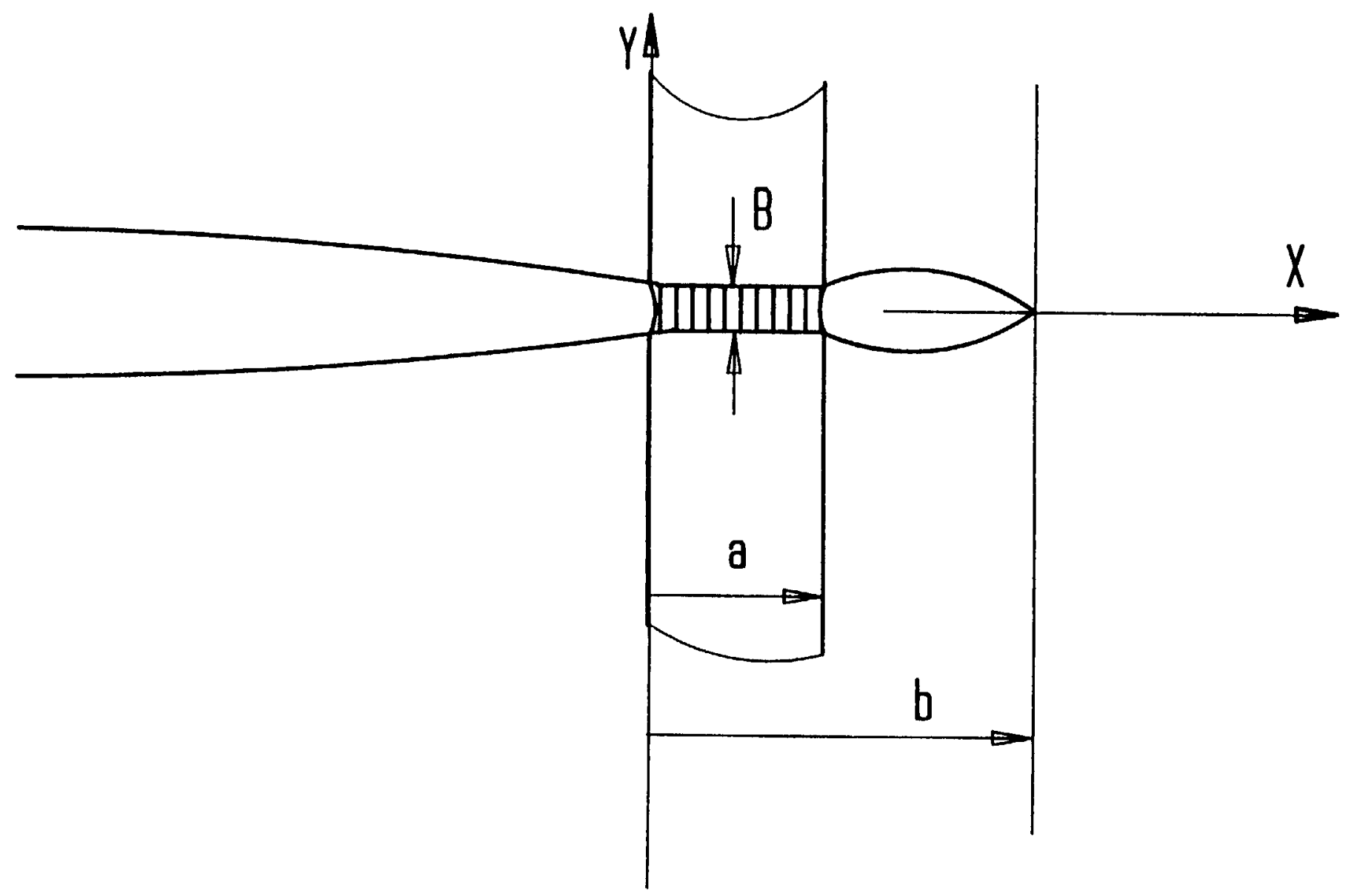

Figure 1.-Single link configuration. 


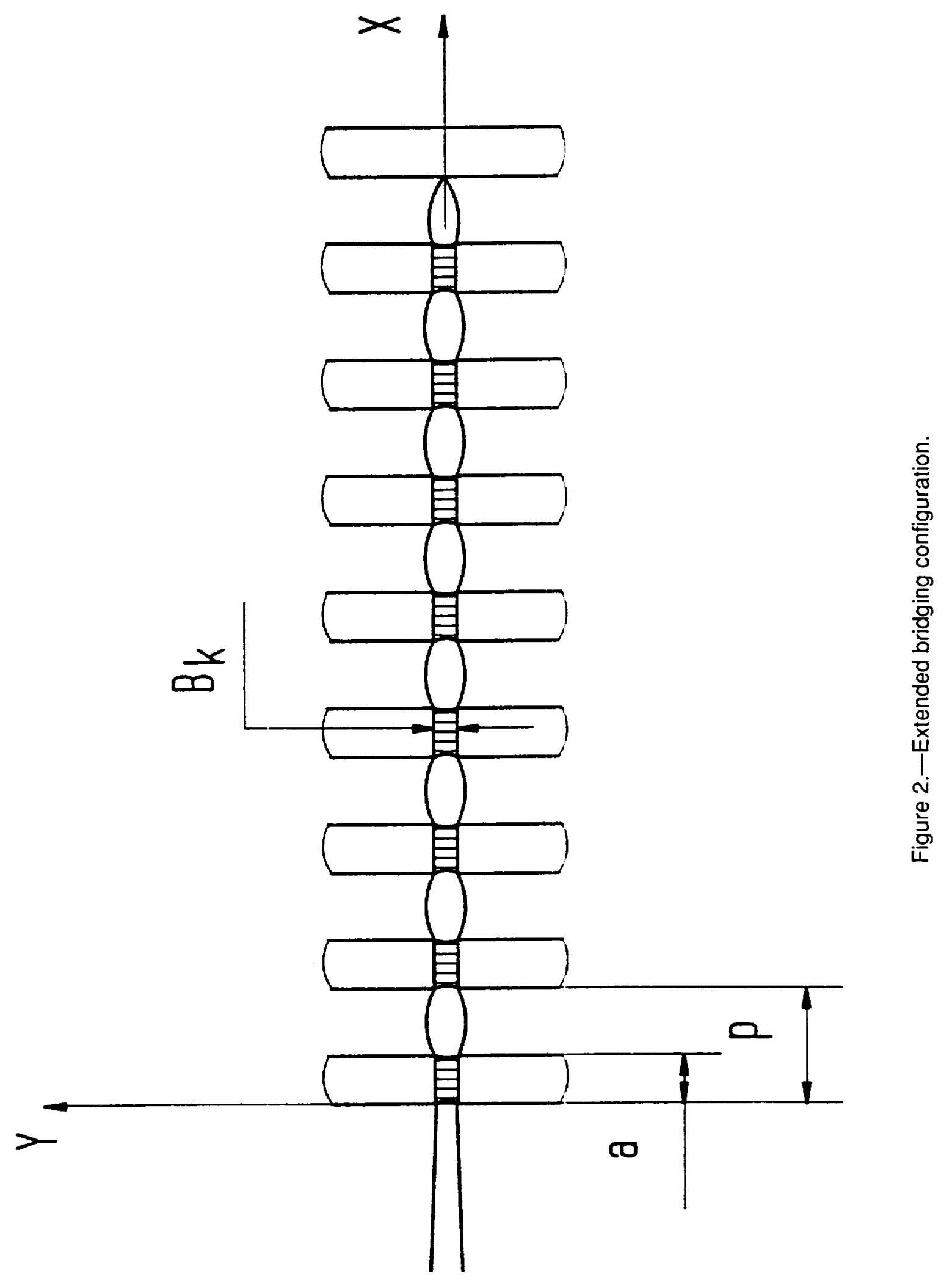



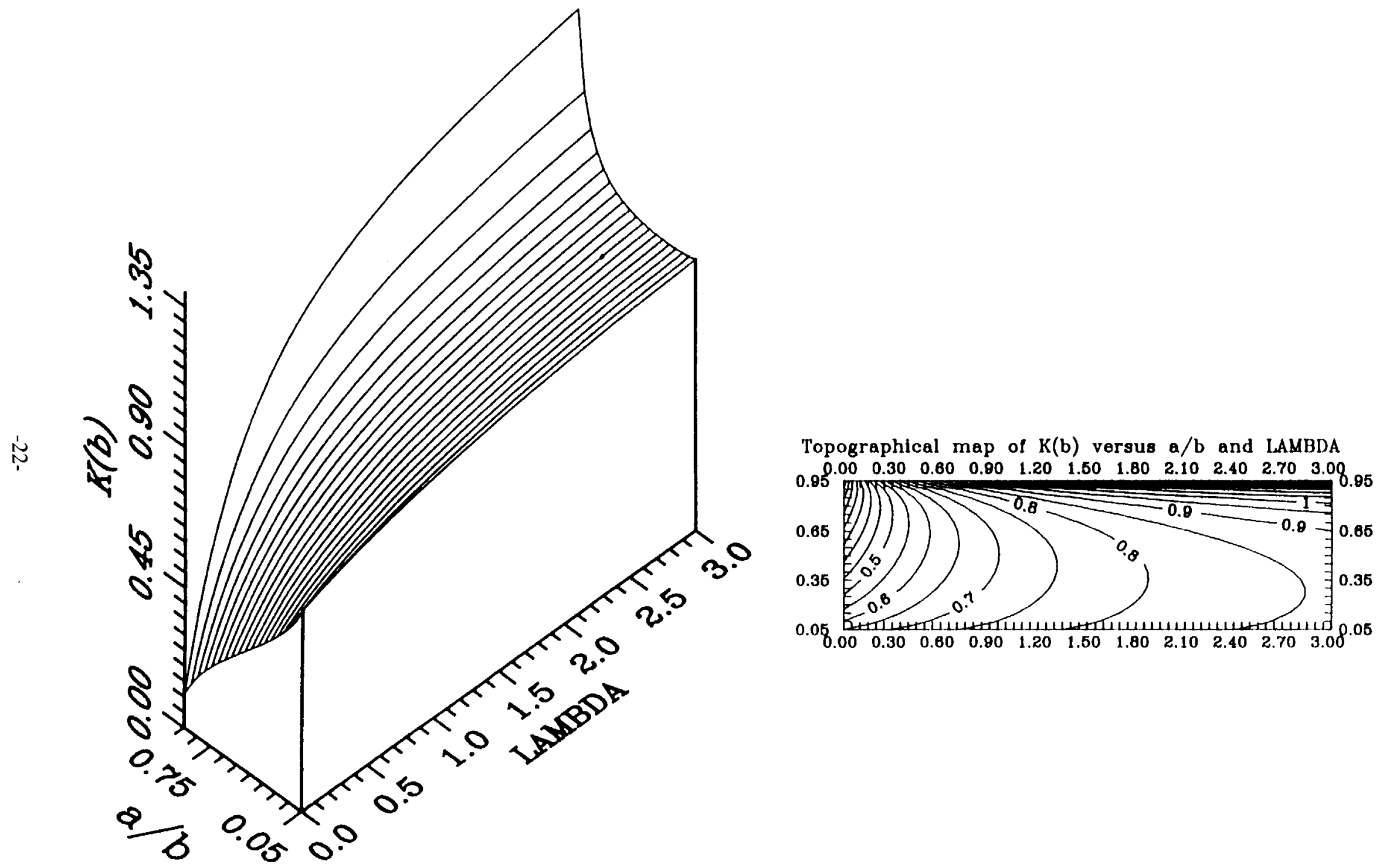

Figure 3.-Stress intensity at the leading crack tip, $x=b$, for the case of the single link model and the corresponding topographical map. The data are given as a function of $a / b$ and $\Lambda$; the values of the stress intensity factor are normalized by $K^{\infty}$. 

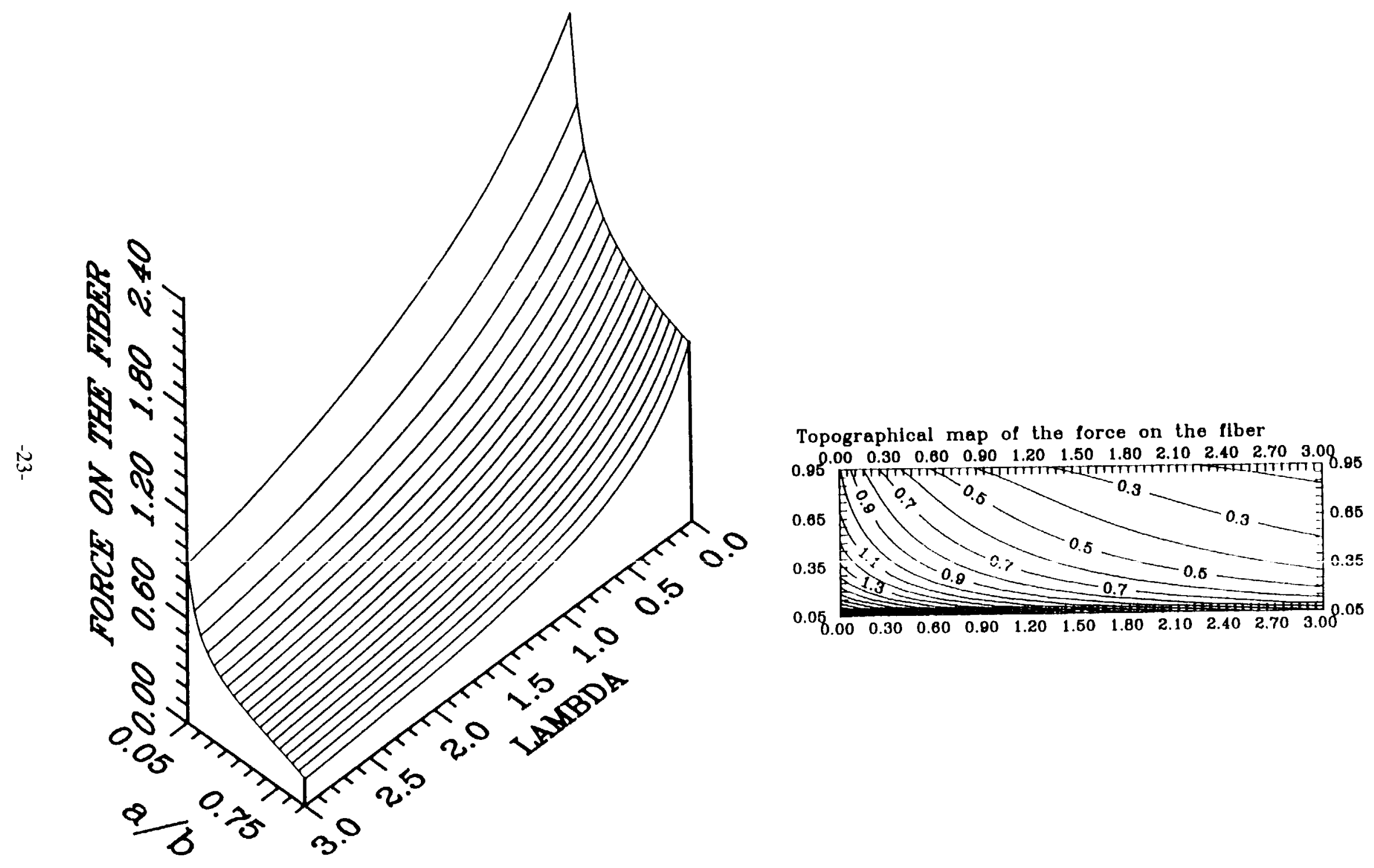

Figure 4.- Net force acting on the fiber for the case of single link model versus the ratio $a / b$ and parameter $\Lambda$ and the corresponding topographical map. The data are normalized by the force acting on the link prior to microcracking, Equation (3.1). 

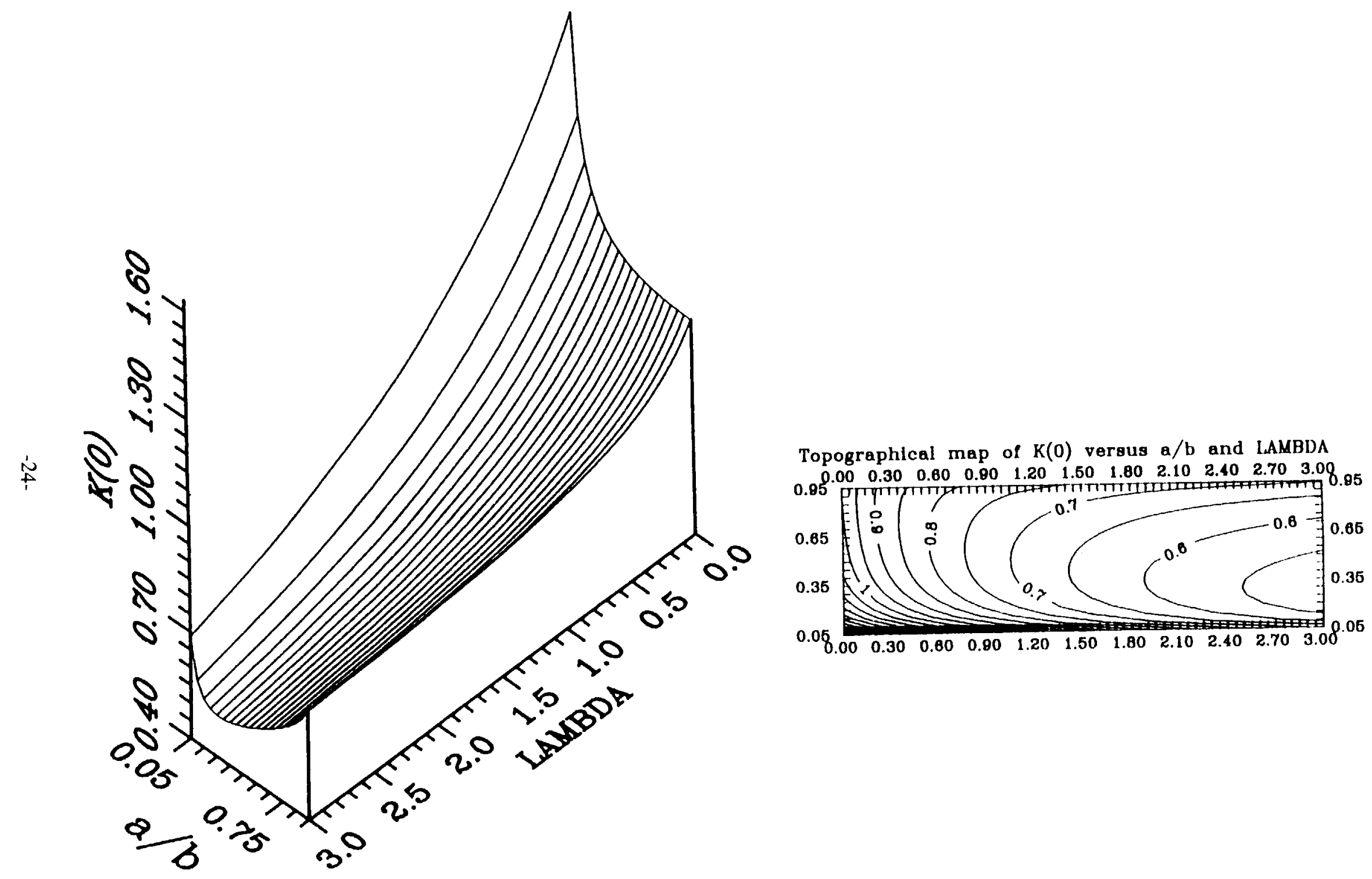

Figure 5. Stress intensity at the main crack tip, $x=0$, for the case of the single link model and the corresponding topographical map. The data are given as a function of $a / b$ and $\Lambda$; the values of the stress intensity factor are normalized by $K^{\infty}$. 

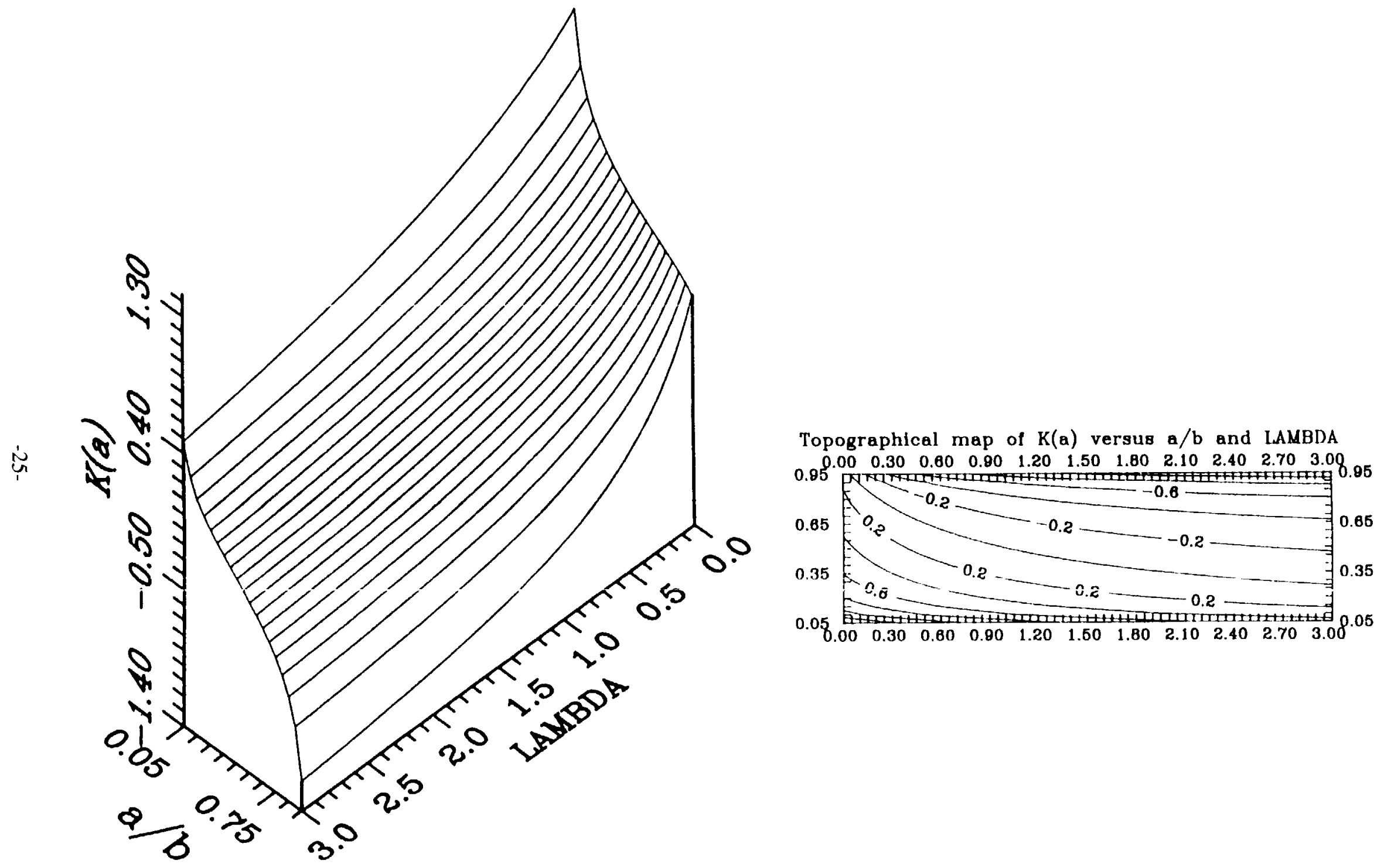

Figure 6.-Stress intensity at the trailing tip of the microcrack, $x=a$, for the case of the single link model, and the corresponding topographical map. The data are given as a function of $a / b$ and $\Lambda$; the values of the stress intensity factor are normalized by $K^{\infty}$. 


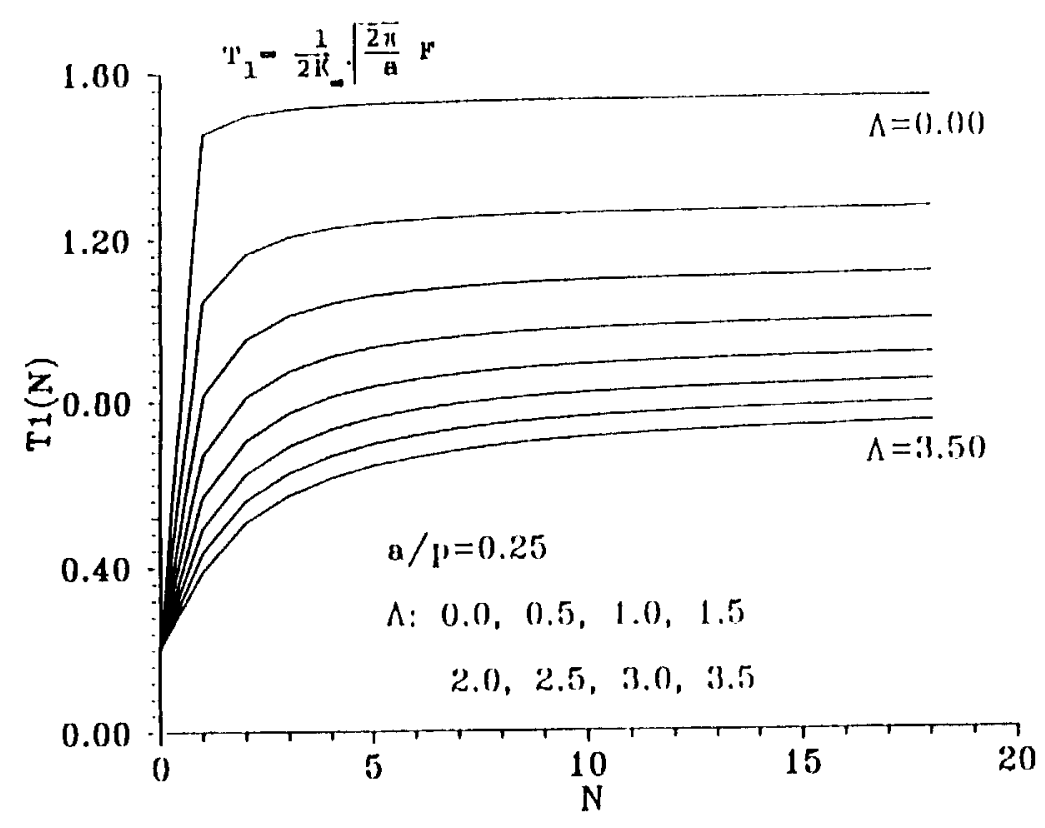

(a) $a / p=0.25$.

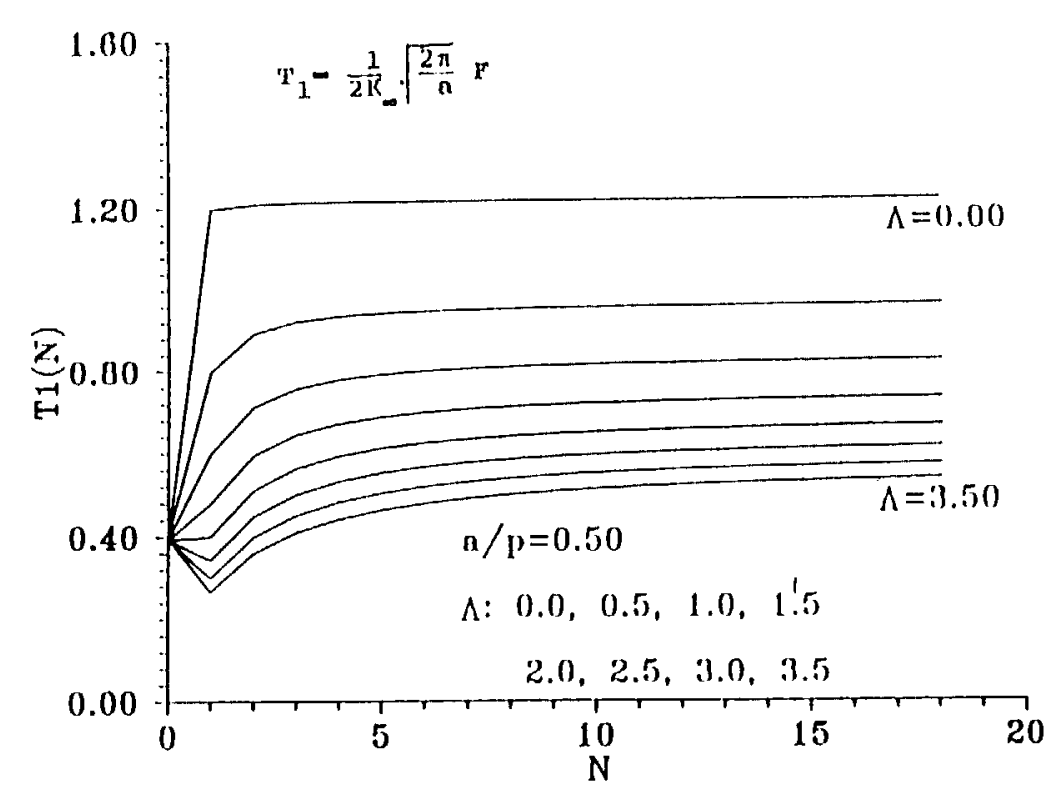

(b) $a / p=0.50$.

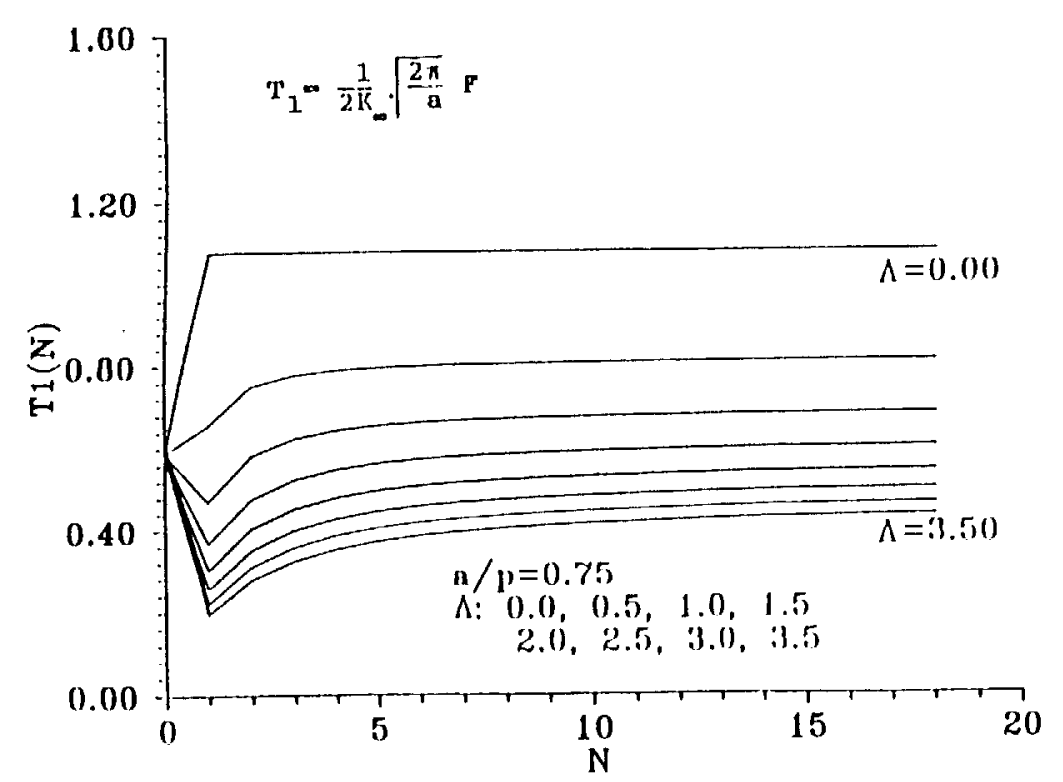

(c) $a / p=0.75$.

Figure 7.-Net traction on the first fiber (trailing end) versus the length of the bridging zone in terms of number of microcracks. For $N=0$, only a portion of the net force acting on the fiber cross-section (assumed circular) is accounted for. 


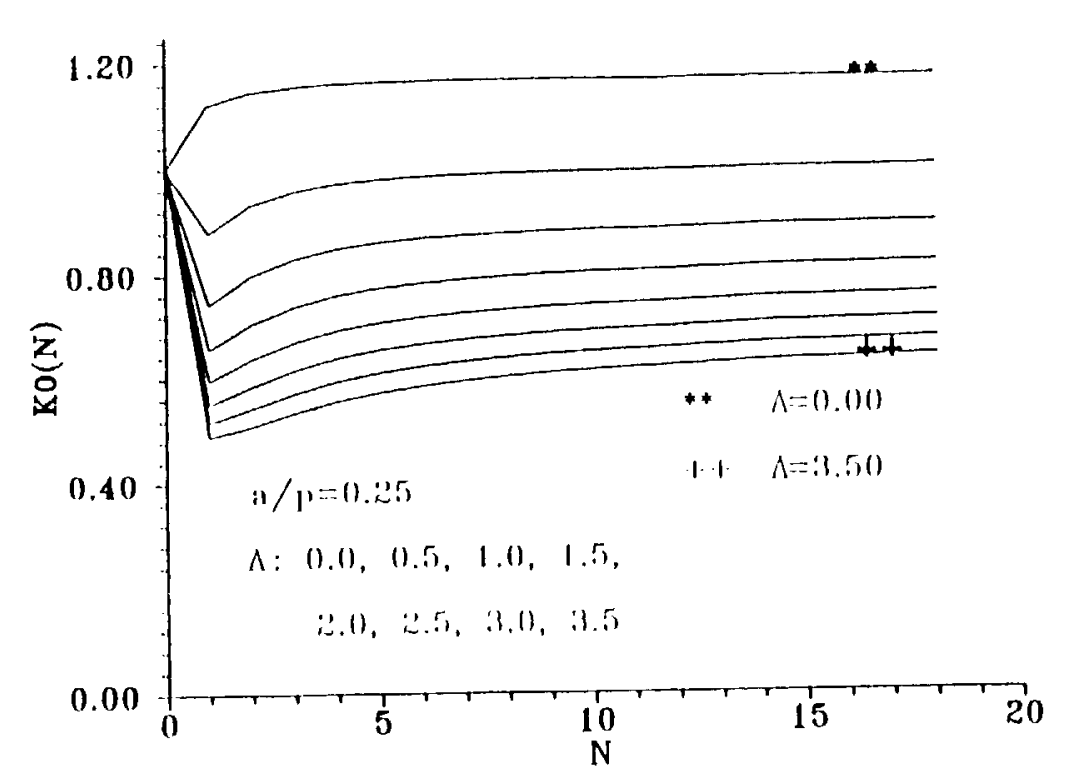

(a) $a / p=0.25$.

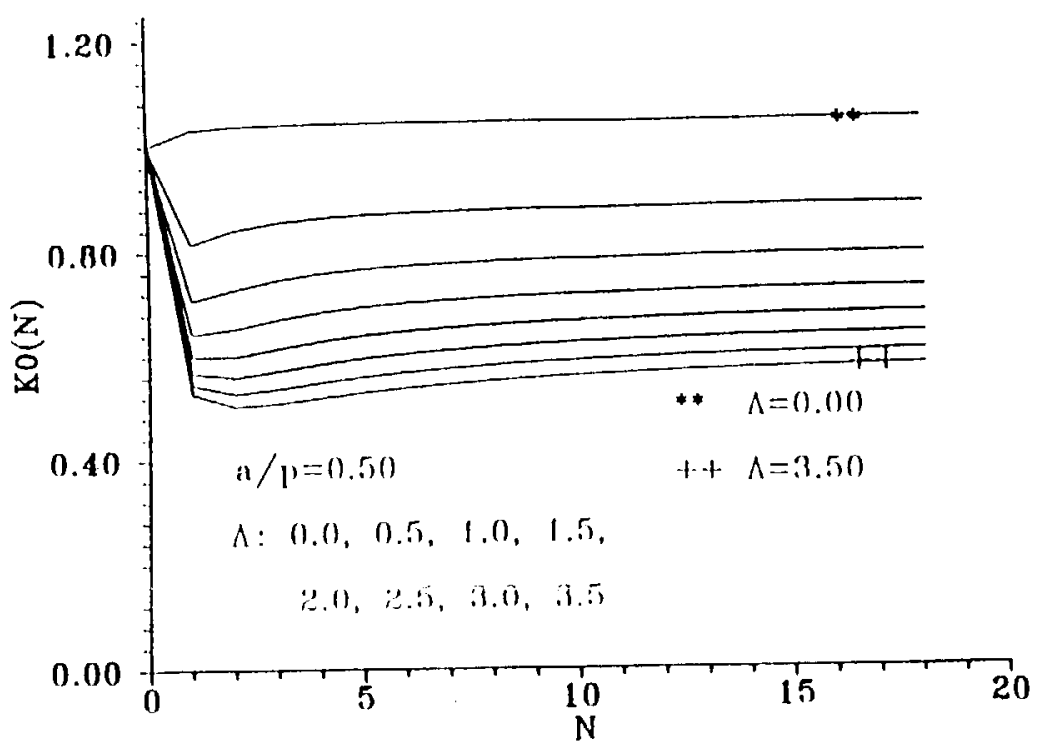

(b) $a / p=0.50$.
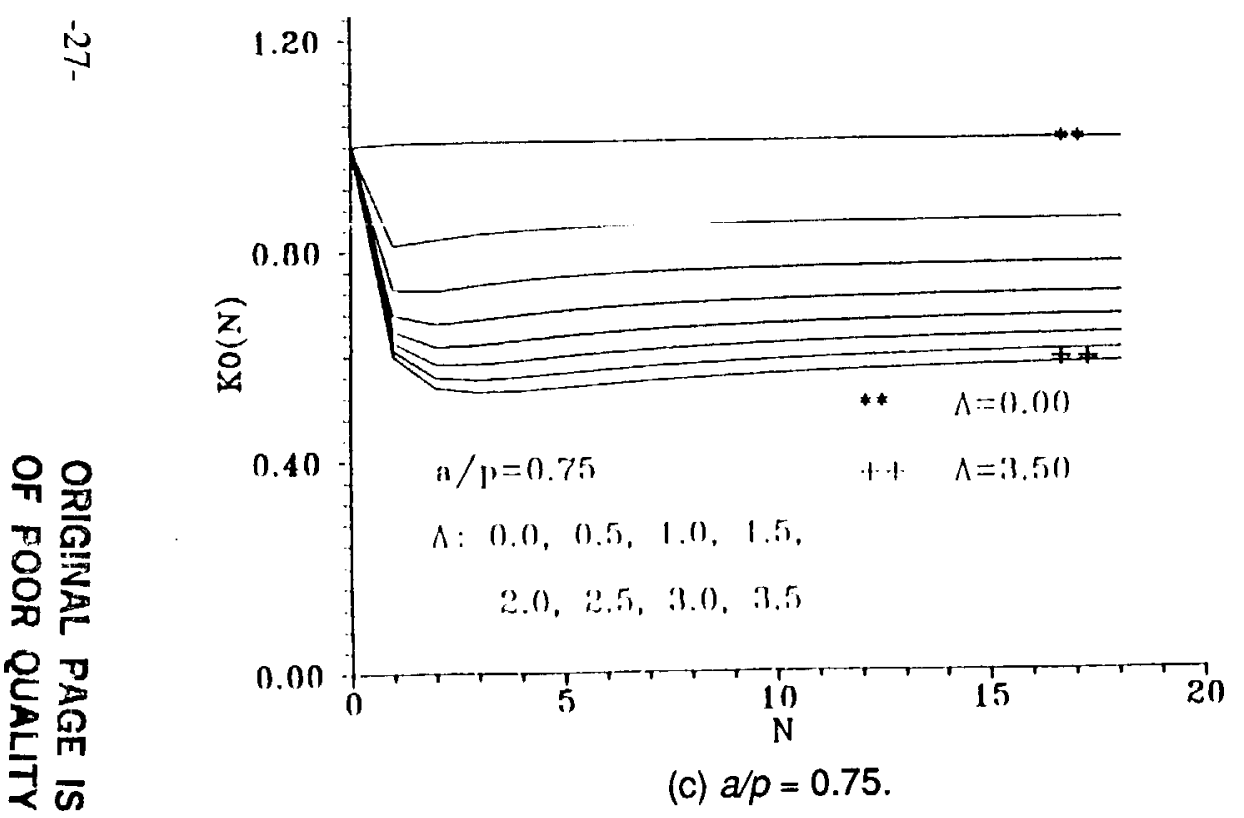

(c) $a / p=0.75$

Figure 8.-Stress intensity factor at the tip of the main crack versus the length of the bridging zone in terms of the number of microcracks in it. The data are normalized by the value of the remote stress intensity factor. 


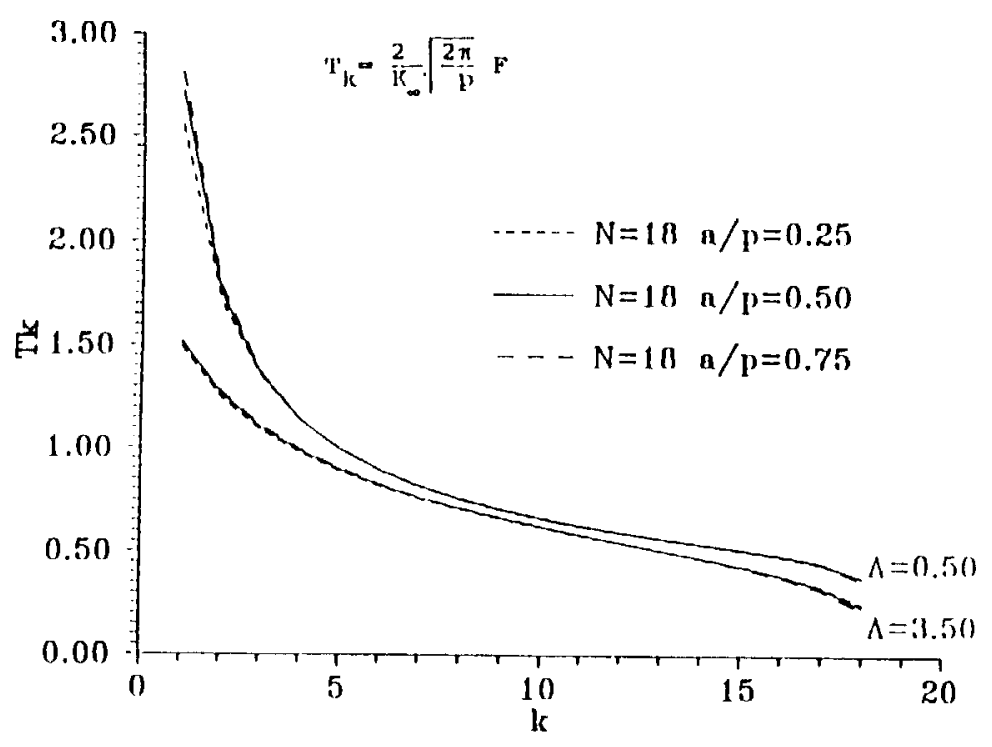

(a) Distribution of the fiber traction.

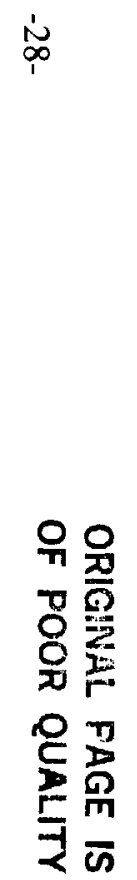

Distribution of stress intensity factors on leading tip of microcracks within bridging zone for $a / p=0.50$.

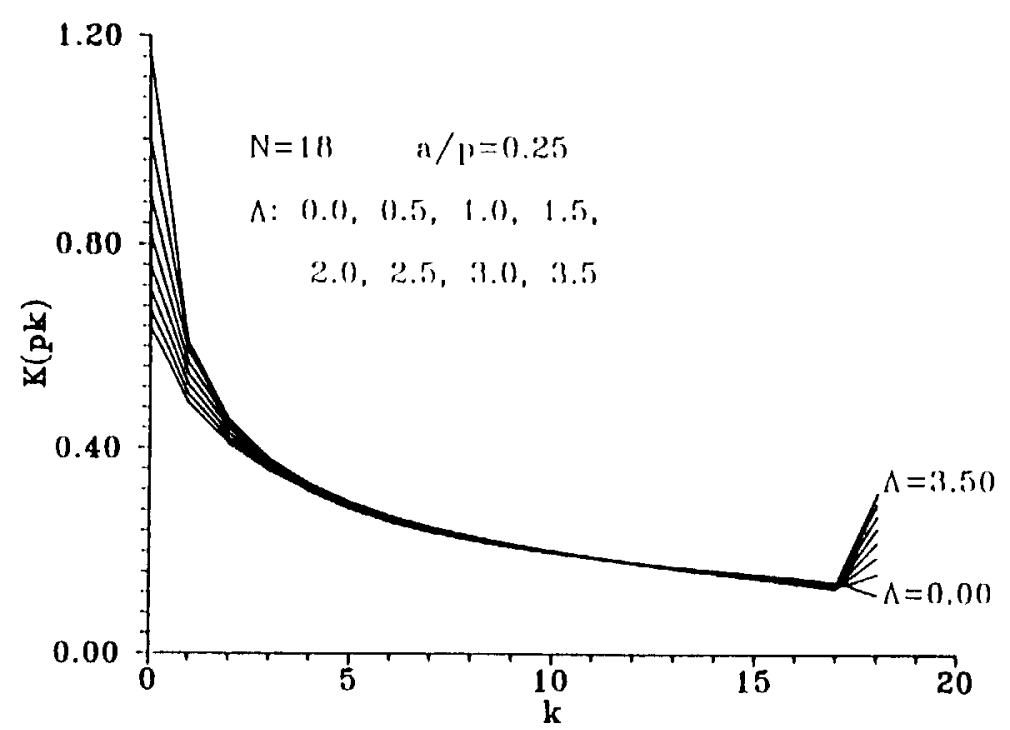

(b) Distribution of stress intensity factors on leading tip of microcracks within bridging zone for $a / p=0.25$.

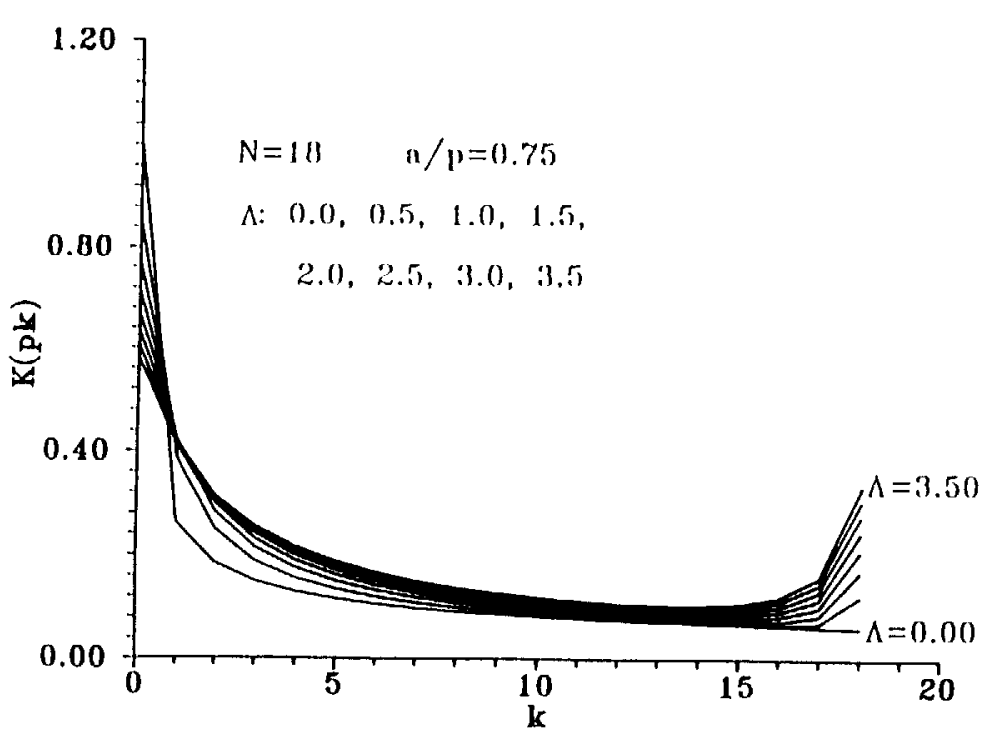

(d) Distribution of stress intensity factors on leading tip of microcracks within bridging zone for $a / p=0.75$.

Figure 9.-Fracture parameters within the bridging zone $N=18, N$ is a number of microcracks in the bridging zone. 


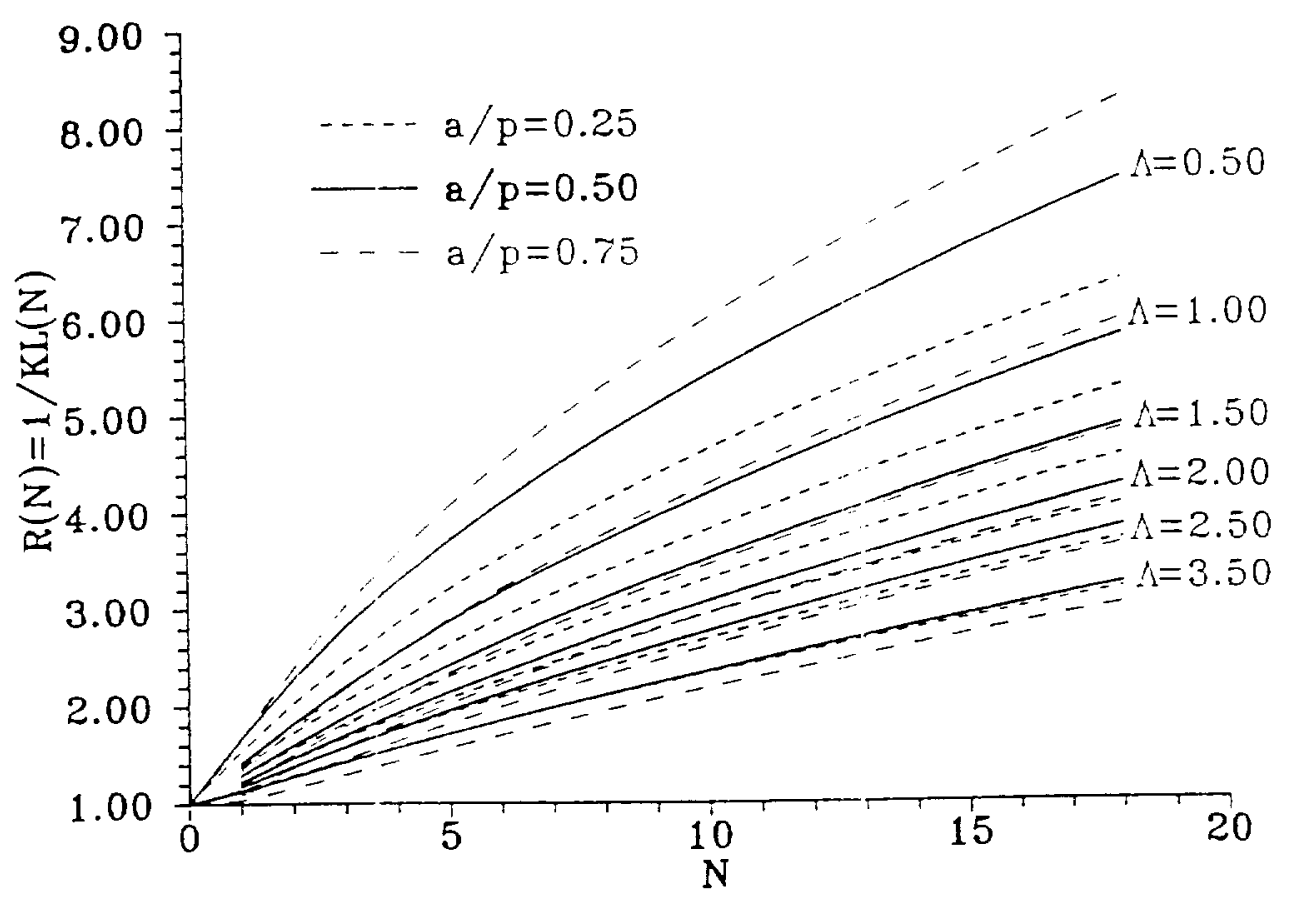

(a) Resistance curves versus number of inicrocracks, cases $a / p=0.25 ; a / p=0.50 ; a / p=0.75$.

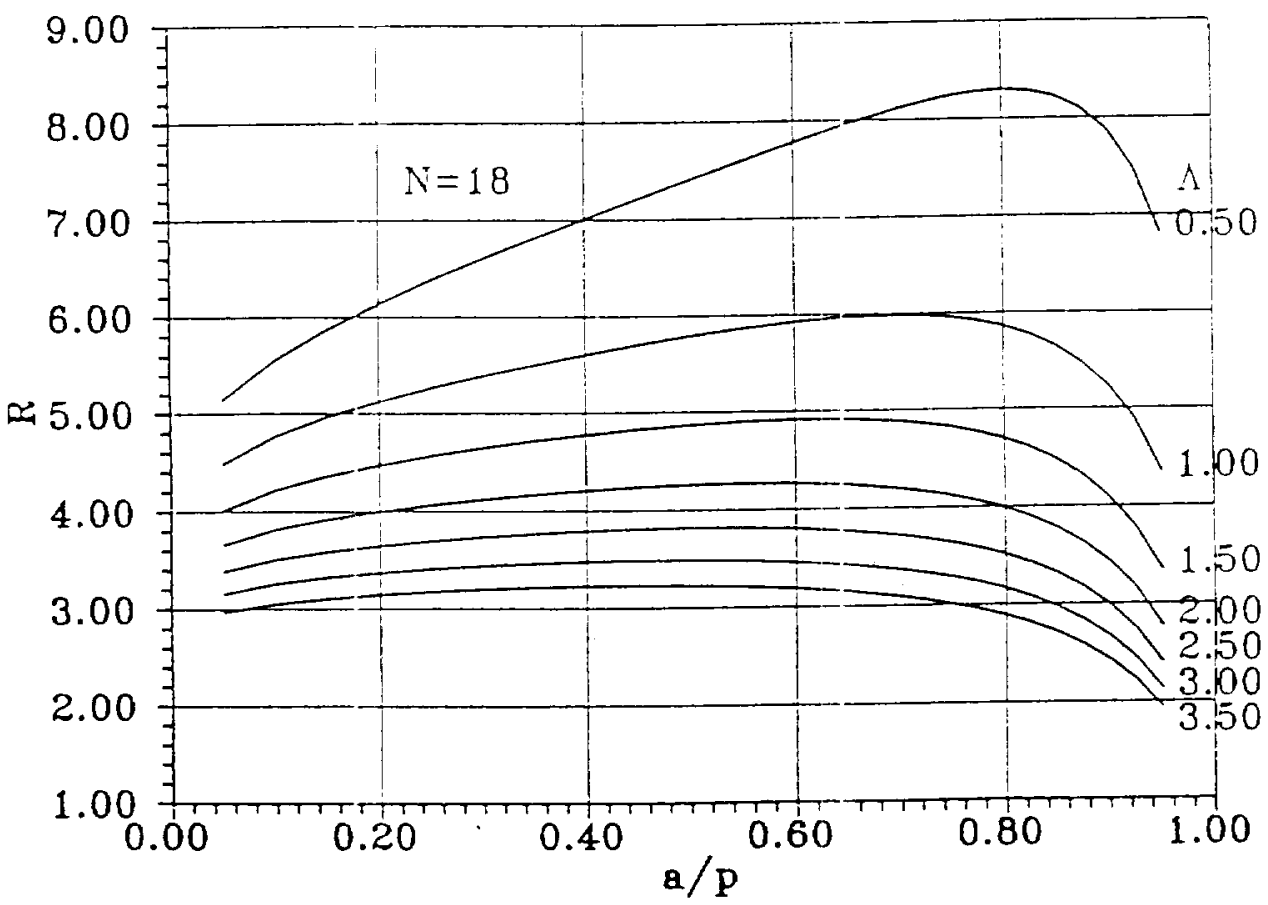

(b) Resistance curves versus fibers spacing ratio $a / p$; the length of the bridging zone constant, $N=18$.

Figure 10.-Fracture resistance curves; bridging zone is given in terms of number of microcracks in it. 


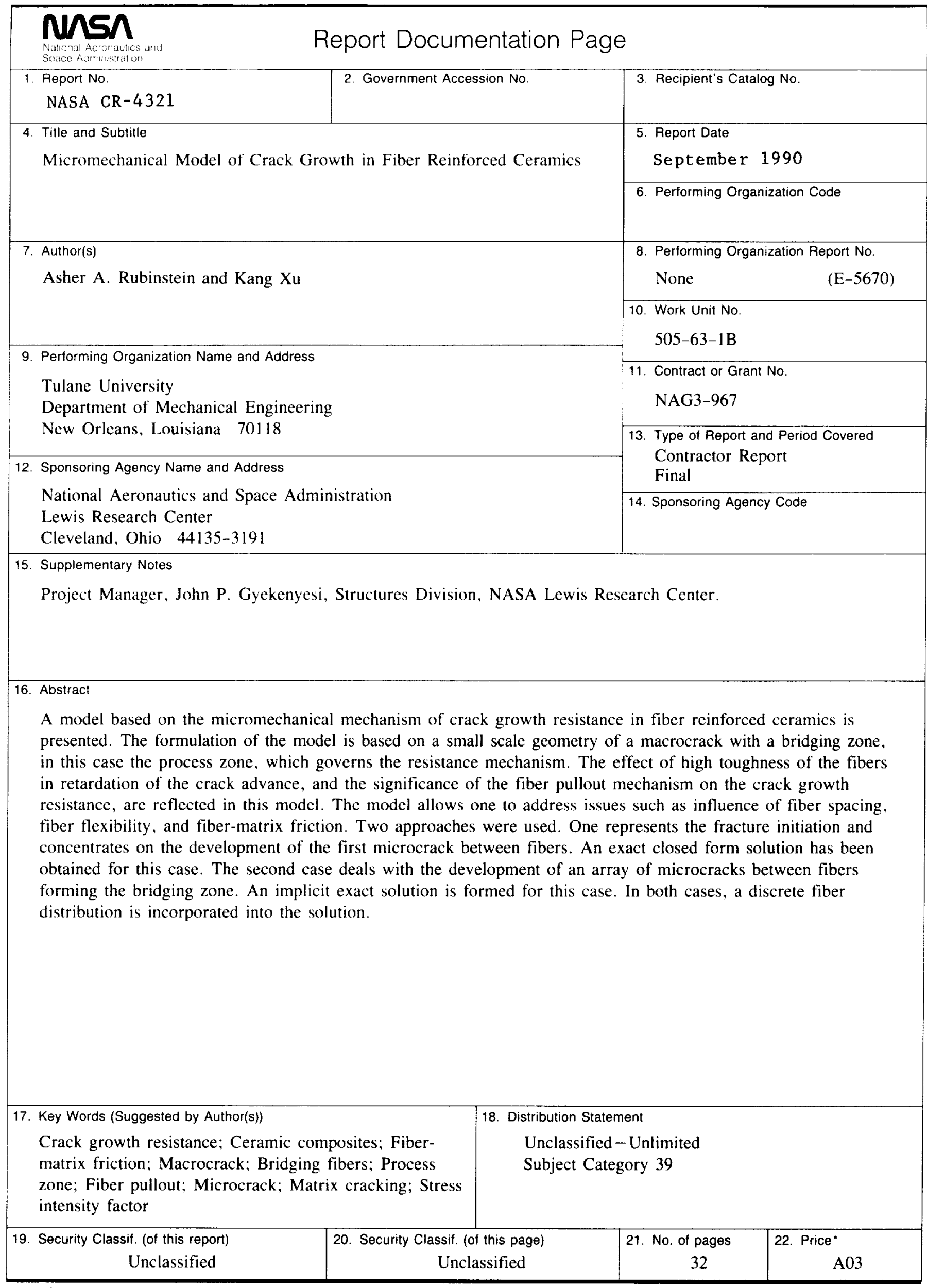




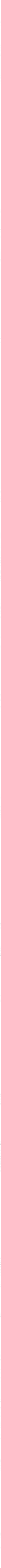

